\title{
FICTION, JOURNAL DE BORD ET RECHERCHE CRÉATION
}

\author{
Éric le Coguiec, Ph.D \\ Universidade de Québec em Montreal \\ E-mail: le_coguiec.eric@uqam.ca
}

Résumé

Instrument de collecte de données, le journal de bord est largement recommandé dans le domaine des sciences sociales, mais aussi de plus en plus suggéré en recherche création. Censé aider le chercheur à réaliser une recherche qui rencontre les critères de scientificité (Savoie-Zajc, 2003, Mucchielli, 1996) certains chercheurs mentionnent toutefois une difficulté de s'astreindre à la consignation de données (Baribeau, 2005). Sans remettre en question l'utilité du journal de bord en recherche création, je propose ici de questionner un outil méthodologique en proposant une conception du journal de bord élargie à la fiction.

\section{Mots-clés}

Recherche Création. Méthodologie de la Recherche. Journal de Bord. Fiction
Resumo

Instrumento de coleta de dados, o diário de campo é amplamente recomendado no campo das ciências sociais, mas também cada vez mais indicado na pesquisa-criação. Supõe-se que esse instrumento metodológico contribua para a realização de uma pesquisa que atenda a certos critérios de cientificidade (Savoie-Zajc, 2003, Mucchielli, 1996). No entanto, alguns pesquisadores apontam dificuldades para se ater à análise e interpretação dos dados (Baribeau, 2005). Sem questionar a utilidade do diário de campo em pesquisa-criação, propõe-se uma concepção ampliada de diário de campo, que se estenda para a ficção.

Palavras-chave

Pesquisa-Criação. Metodologia da Pesquisa. Diário de campo. Ficção 
Instrument de collecte de données, le journal de bord est largement recommandé dans le domaine des sciences sociales, mais aussi de plus en plus suggéré en recherche création. Censé aider le chercheur à réaliser une recherche qui rencontre les critères de scientificité (Savoie-Zajc, 2003, Mucchielli, 1996) certains chercheurs mentionnent toutefois une difficulté de s'astreindre à la consignation de données (Baribeau, 2005). Sans remettre en question l'utilité du journal de bord en recherche création, je propose ici de développer une réflexion sur cet outil méthodologique qui a peu été questionné et de proposer une conception du journal de bord élargie à la fiction.

Dans le film Existenz (1999) du cinéaste canadien David Cronenberg le réel, du moins ce qu'on entend par réel est mis en cause (Marcel, 1999) tant il est difficile, voire impossible, pour les protagonistes du film de distinguer ce qui relève du jeu de la réalité en introduisant notamment les corps dans l'univers des jeux vidéo virtuels. Contrairement aux acteurs qui ne parviennent plus à savoir s'ils sont dans l'espace du jeu ou s'ils sont dans la réalité, le spectateur d'Existenz sait qu'il regarde un film, autrement dit une représentation du monde réel qui l'amène en l'occurrence à réfléchir sur l'obsession de la modernité à développer la technologie au-delà de ses limites, remettant en question les possibilités illimitées de la technique. L'expression être plongée dans un film décrit très bien la situation d'immersion fictionnelle vécue par le spectateur placé dans un état de leurre perceptif qui agit à un niveau pré-attentionnel (Schaeffer, 1999). Cependant la fiction n'est pas synonyme de faux-semblant comme l'explique Schaeffer:

[...] la fiction procède certes à travers des leurres pré-attentionnels, mais son but n'est pas de nous leurrer, d'élaborer des semblants ou des illusions; les leurres qu'elle élabore sont simplement le vecteur grâce auquel elle peut atteindre sa finalité véritable, qui est de nous engager dans une activité de modélisation (1999, p. 199).

Le film par le biais du divertissement élabore une modélisation du réel tout en désignant un outil de prospection qui cherche à explorer des possibles (Cohn, 2001).

Si la fiction dans la sphère artistique procure un plaisir esthétique tout en étant un vecteur de connaissance comme c'est le cas dans Existenz, qu'elle est la place réservée aux usages fictionnels dans la sphère universitaire?

Autant la fiction come objet d'étude constitue un champ de recherche (Goodman, 1968 ; Schaeffer, 1999 ; Metz, 1971) autant l'usage de la fiction comme pratique langagière à l'université est encore largement réservé au domaine des sciences sociales qui plus est principalement dans le milieu universitaire anglo-saxon, comme l'indique le nombre de publications sur le sujet depuis une vingtaine d'années (Bagley et Cancienne 2002 ; Banks 2008 ; Banks and Banks 1998 ; Barone and Eisner 2012 ; Dunlop 2001 ; Iser 1997 ; Knowles and Cole 2008 ; Leavy 2009, 2012 ; Norris 2009 ; Viswesaran 1994 ; Mc Niff 2003). L'usage de la fiction en sciences sociales s'inscrit notamment dans le sillon des revendications des formes d'écriture comme construction socio-historique (Richardson et St Pierre, 2005 ; Denzin et Lincoln 1994, 2000 ; Ellis et Bochner, 1999, 2000, 2003,). Pour ces chercheurs qui participent du paradigme postpositiviste, les régimes d'écriture doivent tenir compte de la subjectivité du chercheur, de la relation entre le chercheur et son objet d'étude (Kulick \& Willson, 1995 ; Lewin \& Leap, 1996) et se distinguent 
ainsi des discours scientifiques prétendument neutres, objectifs, universels. Cela signifie que les savoirs produits par ce type de chercheurs sont historiquement, politiquement et linguistiquement situés et idéologiquement ancrés.

Malgré cette perspective postpositiviste, la recherche création a encore peu prêté attention à la question des pratiques langagières et des différentes théories sociolinguistiques. Pourtant, la recherche création devrait être interpellée par cette question tant penser le travail de création interpelle nécessairement les formes de discours et la place qu'occupe la subjectivité dans le discours. Sans revenir en détail sur les spécificités de la recherche création, il est utile de rappeler la posture médiane ou hybride de ce type de recherche qui articule théorie et pratique.

Longtemps réservé aux seuls théoriciens, le discours sur les pratiques en recherche création est construit par les praticiens chercheurs eux-mêmes, à partir de l'étude de leur propre pratique ${ }^{1}$.

Dans le domaine des arts, les travaux de Valéry et de Passeron ont largement contribué à circonscrire le cadre conceptuel de la recherche création (Gosselin, 2006 ; Laurier, 2004 ; Le Coguiec ; 2006). Valéry au sujet des arts du langage écrit que l'étude de l'œuvre en train de se faire, qu'il nomme poïétique, désigne un objet d'étude à part entière qui mériterait autant d'attention que l'étude de l'œuvre achevée. Passeron (1975) étendra le concept de poïétique de Valéry aux arts en général en distinguant, l'esthétique faisant la promotion des

1 J'englobe sous l'expression «recherche création» les disciplines artistiques tels les arts visuels, la musique, le théâtre, la danse, la littérature mais aussi l'architecture, le design, la mode non par souci de simplification, mais d'une part parce que les frontières disciplinaires s'effritent de plus en plus et d'autre part afin de montrer que toutes ces disciplines ont en commun d'articuler théorie et pratique sciences de l'art qui se consomme, celui des sciences des structures propres de l'œuvre en tant que telle (muséologie, scénographie, filmographie, etc.) et celui de la poïétique faisant la promotion des sciences de l'art qui se fait.

Dans le champ de l'architecture et du design, la recherche création se réclame pour une très large part du cadre théorique et conceptuel de la praxis, de la philosophie pragmatiste (Schön, 1996 ; Boutinet, 1990) et de la pensée complexe (Morin, 1990 ; Le Moigne, 1994). Quelques soient les disciplines, il importe «de ne pas réduire l'acte de projet à la dimension et à la phase créative de l'acte de projet» (Findeli, 2007, p.152), ce qui aurait par conséquence à ses yeux de tourner la partie réflexive du projet de recherche sur le monde intérieur du chercheur, ce qui à mon sens n'est pas le cas. Ce type de recherche menée par des praticiens se caractérise par une double production théorie et pratique, qu'elle est ancrée dans le projet et qu'elle explicite à la fois les conditions d'élaboration du projet et la subjectivité du chercheur. Conduire une recherche création suppose que le chercheur prenne point de départ de la recherche sa pratique - i.e le terrain - afin de développer une démarche réflexive visant à saisir ce que parfois il fait sans être en mesure de comprendre. Cela signifie que le chercheur selon les disciplines problématise parfois l'inconnu (Lancri, 2001).

Contrairement aux approches spéculatives et hypothético-déductives qui proposent de construire spéculativement un cadre théorique à partir de théories existantes et qui procèdent déductivement pour appliquer la théorie sur les données empiriques et ainsi expliquer les phénomènes observés, la recherche création est de type inductive et propose de construire un cadre théorique à partir des données. 
Pour reprendre l'expression de Lancri, les concepts sont travaillés par le faire qui en retour travaillent le dire. Engagé dans sa pratique, le chercheur se livre à un travail réflexif « en action » et interprétatif « hors action » afin d'expliciter des connaissances soient tacites et implicites inscrites dans le faire. Le chercheur fait émerger du sens à partir de ses observations puis vérifie l'adéquation des analyses avec celles-ci. Cette perspective méthodologique résonne bien sûr avec la Grounded Theory conceptualisée par Glauser et Srauss (1967) dans le champ des sciences sociales. Dans pareil contexte de recherche, on mesure toute l'importance que recouvre le journal de bord, instrument tout désigné pour assurer le déroulement d'une démarche réflexive et éviter tout assujettissement de la pratique à la théorie.

\section{Journal de bord et recherche}

Afin de faire émerger une théorie des données empiriques et mettre de côté des pré-jugements, des pré-compréhensions, des connaissances antérieures, le chercheur tient un journal de bord, collige des notes d'atelier portant sur les conditions d'élaboration de son travail, sur ses méthodes de création, sur des éléments techniques mais aussi des notes relatives au domaine affectif sans trop prêter attention aux formes de discours admises à l'université.

Ces traces écrites sont censées constituer des aides mémoires (Schatzman et Strauss, 1973), destinées à être dans un deuxième temps analysées afin de construire un cadre théorique à partir des celles-ci. En règle général, dans la version finale du texte, figurent soit des extraits du carnet soit en annexe la totalité du texte. Le journal de bord est ainsi présenté comme un outil méthodologique indispensable pour assurer la triangulation instrumentale (Mucchielli, 1996).

Pourtant le journal de bord n'est pas exempt de critiques. Baribeau (2005) note « qu'il est difficile et astreignant de tenir un journal de bord » (p. 98). En ce qui me concerne, je relève en me basant sur les journaux de bord tenus par les étudiants un certain nombre de points.

Premièrement je note que la subjectivité du chercheur à l'œuvre dans son activité de création est peu présente dans le journal de bord. Les pensées, les sentiments, les impressions, les émotions du chercheur ne rendent que partiellement compte de l'état de son esprit tant celui parfois se voit contraint de se taire. Pourtant l'engagement du chercheur dans sa création, sa proximité avec l'objet d'étude laisserait entendre des formes variées d'écriture. Or le journal de bord s'inscrit encore trop souvent dans une mythologie scientiste (Van Maanen, 1991).

Deuxièmement, en lien avec le premier point, je note que les habitus du chercheur en recherche création tendent à se calquer encore sur celles du chercheur traditionnel : approches méthodologiques, régimes d'écriture, distance par rapport à l'objet d'étude, etc.

Troisièmement, n'ayant pas à sa disposition des modèles de journaux de bord, le chercheur afin de convaincre le lecteur de la véracité des faits afin de satisfaire les injonctions universitaires, invente parfois des données, truffe son journal d'anecdotes.

Quatrièmement, le chercheur rend très peu compte des conditions d'élaboration du projet et tend importer des concepts et à les appliquer sur les données empiriques. Bref, le 
chercheur est enclin à imaginer des données pour qu'elles épousent le cadre théorique. Or les données devraient être issues des données empiriques. Je suis toujours surpris en lisant les mémoires et les thèses issues de la recherche création de constater le peu de notes entourant les conditions économiques, politiques, sociales, historiques entourant la réalisation du projet.

Cinquièmement le chercheur tend à passer sous silence certaines données qui divergent - ou qu'il pense qu'elles divergent des idées généralement admises par les règles qui régissent le champ au sein duquel il évolue.

En somme, le chercheur qui effectue une recherche création est enclin à adopter les règles dominantes qui régissent le monde universitaire et souscrit à des formes d'écriture prédéterminées. Alors que les notes descriptives, les observations, la description de faits, d'événements, la consignation de conversations, etc. sont désignées comme des données brutes, trop souvent elles n'en ont que l'apparence.

\section{La recherche} création et la fiction

Ces observations, me conduisent à penser que la recherche création gagnerait ainsi à considérer la fiction, non en tant qu'objet d'étude, mais en tant qu'objet pratiqué par le chercheur. Plus spécifiquement la fictionnalisation du journal de bord permettrait au praticien chercheur de penser l'écriture comme activité de recherche (Richardson, 2000). L'usage de la fiction donnerait l'occasion de faire effraction dans un contexte universitaire reconnaissable et prémodélisé -corseté ?- dans le but de le repotentialiser notamment en questionnant les formes d'écriture. La fiction, loin d'être uniquement de nature esthétique, désignerait une ruse pour infiltrer le journal de bord et déconstruire son format. Au lieu de rapporter des faits, de faire savoir; de témoigner pour ne pas oublier, en clair de dire le réel, l'intervention de la fiction dans le journal de bord désignerait à la fois une modélisation créative et un lieu de construction de connaissances.

L'usage de la fiction dans le journal de bord en recherche création renvoie à 6 perspectives :

\section{Recouvrer l'expérience du faire}

La fiction en autorisant le chercheur à suspendre les règles de la réalité de manière provisoire stimule le chercheur à recouvrer l'expérience du faire. Même si les événements relatés sont fictifs, ne correspondant pas par exemple au déroulement exact de l'activité de création, cela ne doit pas signifier pour autant que l'expérience n'est pas réelle (Tisseron, 2005). Ce qui compte après tout c'est d'accéder à nouveau à l'expérience du faire pour être capable de la nommer.

\section{Expliciter les conditions d'élabora- tion du projet de création}

En ayant recours à la fiction, certains chercheurs par le biais de la fiction se sentiraient plus enclin à expliciter les conditions d'élaboration de leur projet de création et de porter au jour les soubassements historiques, culturels et sociaux de leurs pratiques. Cet exercice d'explicitation habituellement réservé au sociologue, serait effectué ici depuis l'intérieur même de la pratique. Par le biais de la fiction, le chercheur se sentirait affranchit des contraintes propres au champ et pourrait déconstruire un certain nombre de croyances en indiquant 
pour commencer que l'activité de création n'est pas une activité mystérieuse, que tout projet de création n'est en aucun absolu mais relatif, que celui-ci occupe enfin une place historique. Bien sûr, cette activité de déconstruction via la fiction implique parallèlement un travail d'autodéconstruction de la part du chercheur.

Cet exercice de mise au jour via la fiction permettrait de comprendre l'histoire du champ et de l'espace des possibles dans lequel elle se survit pour reprendre la terminologie de Bourdieu (1998).

\section{Prendre une distance à l'égard d'une forme d'écriture «neutre»}

La création a longtemps été exclue de la sphère universitaire, seul le discours sur les œuvres, avait droit de cité. Pas étonnant que la recherche sur les œuvres et les formes d'écriture qui lui sont associées dominent encore la recherche création. La fiction remettrait en question un régime d'écriture dominant qui loin d'être neutre et universel est lui le produit d'une construction sociohistorique et le fruit d'une lutte entre les acteurs. Comme l'exprime clairement Bourdieu (1991, p. 12)

(...) bien qu'il ait ses propres lois, transcendantes aux consciences et aux volontés individuelles, l'héritage culturel, qui existe à l'état matérialisé et à l'état incorporé (sous la forme d'un habitus fonctionnant comme une sorte de transcendantal historique), n'existe et ne subsiste effectivement (c'est-àdire en tant qu'actif) que dans et par les luttes dont les champs de production culturelle (champ artistique, etc.) sont le lieu, c'est-à-dire par et pour des agents disposés et aptes à en assurer la réactivation continuée.

La fiction constituerait une ruse pour s'affranchir d'une forme d'écriture canonique et permettrait de faire une pratique langagière qui épouserait les contours de la pratique.

\section{Favoriser une mise à distance avec} l'objet d'étude

L'écriture du journal de bord élargie à la fiction favoriserait une démarche réflexive en favorisant une mise à distance avec l'objet d'étude nécessaire pour effectuer le travail de théorisation. Plongé dans la fiction, le chercheur est conduit à suspendre tout jugement, nommer ce qu'il n'est en mesure toujours de comprendre. Délaissant les conduites habituelles, les exigences institutionnelles, les théories existantes, le chercheur ne cherche pas à analyser son objet d'étude dont le sens bien souvent n'émerge que dans l'après coup (Lancri, 2001). Il écrit sans réellement suivre un plan, sans poursuivre de but précis, mettant de côté les buts qui s'était fixés et accueillant les données qui se présentent à lui sans le rejeter. Par la fiction, le chercheur parvient à prendre une distance avec le faire et se traiter lui-même comme un autre (Ricœur, 1990).

\section{Recouvrer la langue parlé ou verna-} culaire

Le chercheur en s'immergeant dans la fiction peut s'autoriser à écrire à la marge des normes langagières recommandées par le milieu universitaire. II peut par exemple recouvrer la langue de ses origines, dont il a toujours fait usage et dont il a été contraint de mettre de côté lorsqu'il a franchi l'enceinte universitaire pour adopter la « langue de la norme » expression d'autres habitus. Cette conversion partielle de l'habitus originaire est souvent le prix à payer pour entrer dans un nouveau champ et la langue n'y échappe pas comme l'exprime avec justesse Eribon (2009) dans son livre $R e-$ 
tour à Reims. L'auteur explique que pour accéder au statut de professeur à l'université, il fut contraint de désapprendre le langage propre du milieu populaire dont il est issu et taire ses origines sociales.

Réapprendre à parler fut tout autant nécessaire: oublier les prononciations et les tournures de phrase fautives" les idiomatismes régionaux (ne plus dire qu'une pomme est « fière ", mais qu'elle est « acide »), corriger l'accent du Nord-Est et l'accent populaire en même temps, acquérir un vocabulaire plus sophistiqué, construire des séquences grammaticales plus adéquates... bref, contrôler en permanence mon langage et mon élocution (p. 81).

Eribon confirme ici les thèses de Bakhtine (1977), Bernstein (1990) et Labov (1972) qui montrent que le langage n'est pas un système autonome instituant se développant en lui-même mais qu'il existe une relation entre la structure de la langue et la structure sociale.

Le sens du mot est entièrement déterminé par son contexte. En fait, autant de contextes, autant de significations possibles. Néanmoins, le mot ne cesse pas pour autant d'être un. II ne se désagrège pas en autant de mots qu'il existe de contextes où il peut s'insérer. Bien entendu, cette unicité du mot n'est pas seulement assurée par l'unicité de sa composition phonétique, il y a aussi unicité inhérente à toutes ses significations. Comment concilier la polysémie du mot érigée en principe et son unicité (Remysen, 2011) ? C'est ainsi que nous pouvons formuler, grossièrement et de façon élémentaire, le problème fondamental de la sémantique. (Bakhtine, 1977, p. 11)

Les usages du langage sont ainsi toujours contextualisés et situés physiquement, institutionnellement et historiquement. User de la langue vernaculaire dans le contexte universi- taire est encore souvent perçu, du moins l'extérieur des sciences sociales, comme l'expression d'une structure sociale inférieure. $Y$ avoir recours désignerait un acte de résistance et la fiction une stratégie pour faire effraction. Mais pas seulement, cela permettrait aussi de renouer avec l'altérité, c'est-à-dire avec ce qui n'est pas considéré comme une pratique « normale ».

\section{Exprimer une subjectivité qui défie les jugements dominants}

L'existence d'une relation entre la structure de la langue et la strate sociale, ne doit pas occulter l'existence d'une relation entre la structure de la langue et son rôle dans la construction des identités sociales et individuelles (Leap, W. \& Boellstorff, 2004). Même si le paradigme postpositiviste autorise les chercheurs à ne pas gommer les positionnements identitaires et à les intégrer dans leur recherche (Rabinow, 1977 ; Tedlock, 1992, Leap, 2004) force est de constater que la prise en compte de l'articulation entre genre, sexualités et langage varie selon les disciplines et les contextes culturels (Greco, 2014 ; Boutet et Mainguenau, 2005). S'il est possible d'assumer son positionnement identitaire en recherche création, l'absence d'études sur ce sujet limite les chercheurs à questionner la dimension idéologique intrinsèque à la vision binaire du genre.

Des analyses des pratiques langagières ont montré que la communauté gay pour résister aux processus de normalisation et de régulation des genres et des sexualités de l'ordre dominant et résister à la marginalisation sociale, a créé diverses stratégies langagières (Hayes 1981 ; Leap 1996 ; Baker 2002 ; Boellstorff 2004). La communauté gay afro-américaine a ainsi re-signifié le lexique (Johnson 2004). En 
Indonésie les homosexuels parlent ce qu'ils appellent le « bahasa gay» langage gay », un phénomène linguistique basé sur la langue officielle de I'Indonésie (Boellstorff, 2004). Cette réinvention de la parole de nature politique en ce qu'il permet de se réapproprier par le langage un espace d'action (Greco, 2014) crée des structures de sociabilité et d'échange.

De Rimbaud à Burroughs la littérature regorge d'exemples de création de nouvelles formes linguistiques qui interrogent la construction et la déconstruction du genre et des sexualités. Rappelons que Rimbaud pour échapper dans la société du Second Empire et de la Troisième République aux restrictions d'ordre judiciaire ainsi que la pathologisation qui frappait alors de plein fouet la sexualité de même sexe, développa la métaphore comme tactiques de diversion / subversion pour traiter de signifiants interdits (Max Kramer, 2011).

Je ne sous-entends pas ici que la recherche création rejette tout positionnement identitaire, mais l'absence d'études reliant la recherche création, les études sur le genre et la sociolinguistique ne favorise pas des formes d'écriture qui prennent en compte la construction identitaire du chercheur. Or le langage pour certains d'entre eux constitue une tactique de resubjectivation (De Certeau, 1990, Bucholtz et Hall, 2004 ; Éribon, 2012).

\section{Conclusion}

L'utilisation du journal de bord comme instrument de recherche a peu fait l'objet d'étude alors qu'il fait partie des instruments méthodologiques en recherche création. La crise d'une représentation objective du savoir a pourtant ouvert la voie à des formes d'écriture variées -créatives- qui autoriserait pourtant ce type questionnement. Étant donné l'engagement du chercheur dans ce type de recherche, il importerait de considérer davantage des formes d'écriture qui rendent compte de cette implication.

Loin de favoriser l'activité réflexive du projet de recherche sur le monde intérieur du chercheur, l'émergence de formes d'écriture dynamiserait au contraire la recherche sur le monde extérieur, c'est-à-dire sur les conditions d'élaboration du projet de création encore trop souvent absentes des travaux de recherche. La fiction présente dans l'activité de création devrait ainsi se manifester dans l'activité de recherche et le journal de bord semble un lieu propice pour qu'elle œuvre. Pour le moment, l'usage de la fiction doit être pensé non comme production textuelle finale, mais comme une stratégie langagière pour théoriser la pratique. La capacité de la fiction à ordonner les événements dans une trame signifiante, en fait un formidable levier de construction du sens (Weick, 1995). II est clair que tout chercheur doit bien sur faite preuve d'une responsabilité éthique et informer le lecteur de la tout passage fictif du texte. Comme le souligne Schaeffer (1999), il importe de respecter le « contrat de la fiction ». Le lecteur doit être capable de distinguer ce que je produis ou perçois en tant que fiction. Ce contrat qui varie en fonction des cultures et des contextes, constitue est une des conditions de la phylogenèse de la fiction : son ancrage social.

Referências

AÏT-TOUATI, F. Contes de la lune: essai sur la fiction et la science modernes. Paris: Gallimard, 2011. 
BAGLEY, C., \& CANCIENNE, M. B. Educational research and intertextual forms of (re)presentation: The case for dancing the data. Qualitative Inquiry, v. 2, n. 7, 2001.

BAGLEY, C.; CANCIENNE, M. B. (eds.). Dancing the data. New York: Peter Lang, 2002.

BAKER, P. Polari-the lost language of gay men. Londres: Routledge, 2003.

BAKHTINE, M. Le Marxisme et la philosophie du langage: essai d'application de la méthode sociologique en linguistique. Paris, Éd. de Minuit, 1977.

BANKS, A.; BANKS, S. P. (eds.). Fiction and social research: By ice or fire. Walnut Creek, CA: AltaMira Press, 1998.

BANKS, S. Writing as theory. Handbook of the arts in qualitative research. Londres: Sage, 2008.

BARIBEAU, C. L'instrumentation dans la collecte de données. Recherches Qualitatives. Hors-série, n. 2, p. 98-114, 2005.

BARONE, T.; EISNER, E. W. (eds.). Arts based research. Londres: Sage, 2011.

BERNSTEIN, B. The Structuring of Pedagogical Discourse. London: Routledge, 1990.

BOCHNER, A. P; ELLIS, C. S. Which way to turn?. Journal of Contemporary Ethnography, v. 28, n. 5, p. 485-499, 1999.
BOELLSTORFF, T. Gay language and Indonesia: Registering belonging. Journal of Linguistic Anthropology, v.14, n. 2, p. 248-268, 2004.

BOURDIEU, P. Le champ littéraire. In: Actes de la recherche en sciences sociales, v. 89, p. 3-46, setembro, 1991.

BOURDIEU, P. Les Règles de l'art. Genèse et structure du champ littéraire. Paris: Seuil, 1998.

BOUTET J.; MAINGUENEAU, D. Sociolinguistique et analyse de discours: façons de dire, façons de faire. Langage et société, v. 114, n. 4, p. 15-47, 2005.

BOUTINET, J. Anthropologie du projet. Paris, PUF,1990.

BUCHOLTZ, M., HALL, K. Language and identity. In DURANTI, A. (ed.) A Companion to Linguistic Anthropology. Malden: Blackwell, 2004, pp 268-294.

COHN, D. Le propre de la fiction. Paris: Seuil, 2001.

DE CERTEAU, M. L'invention du quotidien. Paris: Gallimard, 1990.

DENZIN, N. K.; LINCOLN, Y. S. The discipline and practice of qualitative research. In DENZIN, N. K.; LINCOLN, Y. S. (ed.). Handbook of qualitative research, 2000, p. 1-28.

DUNLOP, R. Excerpts from Boundary Bay: A novel as educational research. In NEILSEN, L; A. L. COLE; J. G. (eds.), The art of writing inquiry, Halifax: Backalong Books, 2001, p. 1125. 
ELLIS, C. The ethnographic I: A methodological novel about autoethnography. Walnut Creek, CA: AltaMira Press, 2004.

ELLIS, C. S.; BOCHNER, A. (2000). Autoethnography, personal narrative, reflexivity: Researcher as Subject. Communication Faculty Publications. Paper 91, 2000.

ELLIS, C. S.; BOCHNER, A. P. An introduction to the arts and narrative research: art as inquiry. Qualitative Inquiry, v. 9, n. 4, p. 506-514, 2003.

ERIBON, D. Retour à Reims: Une théorie $d u$ sujet. Paris: Fayard, 2009.

ERIBON, D. Réflexions sur la question gay. Paris: Flammarion, 2012.

FINDELI, A., \& COSTE, A. De la recherchecréation à la recherche-projet: un cadre théorique et méthodologique pour la recherche architecturale. Lieux communs, n. 10, p. 139161, 2007.

FLOWER, L.; HAYES, J. R. A cognitive process theory of writing. College composition and communication, v. 32, n. 4, p. 365-387, 1981.

GLASER, B.G.; STRAUSS, A.L. The Discovery of Grounded Theory: Strategies for Qualitative Research. Chicago, IL : Aldine, 1967.

GOODMAN, N. Languages of art: An approach to a theory of symbols. Indianapolis: Hackett publishing, 1968.
GOSSELIN, P.; LE COGUIEC, E. La Recherche création. Pour une compréhension de la recherche en pratique artistique, Québec, Presses de l'Université du Québec, 2006.

GRECO, L. Les recherches linguistiques sur le genre: un état de l'art. Langage et société, n. 2, p. 11-29, 2014.

HAYES, JJ. Lesbians, gay men and their languages. In J. W. CHESEBRO (ed.), Gayspeak. New York: The Pilgrim Press, 1981, p. 28-42.

HERSKER, A. L.; LEAP, W. Representation, Subjectivity and Ethics in Urban Gay Ethnography. City \& Society, n. 12, p.142-147, 1996.

ISER, W. (1997). The Use of Fiction in Literary and Generative Anthropology: An Interview With Wolfgang Iser Anthropoetics, v. 3 n. 2, 1997/1998.

JOHNSON, P. Mother Knows Best: Black Gay Vernacular and Transgressive Domestic Space. In W. LEAP, W.; T. BOELLSTORFF (eds.). Speaking in Queer Tongues. Globalization and Gay Language. Champaign: University of Illinois Press, 2004, p. 251-278.

KNOWLES, J. G.; COLE, A. L. Handbook of the arts in qualitative research: Perspectives, methodologies, examples, and issues. Londres Sage, 2008.

KRAMER, M. Pour une poétique queer de Rimbaud. Itinéraires, 2011-1/2011, p. 123-136, 2011. 
KUHN, T. S. The structure of scientific revolutions. Chicago: University of Chicago Press. 1996.

LABOV, W. Sociolinguistic patterns, n. 4. University of Pennsylvania Press, 1972.

LANCRI, J. Modestes propositions sur les conditions d'une recherche en arts plastiques à l'Université. Plastik. n. 1, Editions de la Sorbonne, 2001.

LAURIER, D.; GOSSELIN, P. (eds.). Tactiques insolites: vers une méthodologie de recherche en pratique artistique. Montréal: Guérin, 2004.

LE MOIGNE, J. L. La théorie du système général: théorie de la modélisation. Paris: Dunod, 1994.

LEAP, W.; BOELLSTORFF, T. (eds.) Speaking in queer tongues: Globalization and gay language. Chicago : University of Illinois Press, 2004.

LEAVY, P. Fiction and the feminist academic novel. Qualitative Inquiry, v.18, n.6, p. 516-522, 2012.

LEAVY, P. Low-Fat Love: Expanded Anniversary Edition. Rotterdam: Sense Publishers, 2015.

LEAVY, P. Method meets art: Arts-based research practice. New York: Guilford Publications, 2015.

LEAVY, P. Essentials of transdisciplinary research: Using problem-centered methodologies. Londres : Routledge, 2016.
LEWIN, E.; LEAP, W. Out in the Field. Chicago : University of Illinois Press, 1996.

MAINGUENAU, D. Nouvelles tendances en analyse du discours. Paris: Hachet, 1987.

MARCEL, J. L'a-réalité virtuelle. Ouvrage recensé: eXistenZ de David Cronenberg. 24 images, n. 97, p. 50-51,1999.

MCNIFF, K. On creative writing and historical understanding. Journal of Applied Arts \& Health, v.4, n.1, 29-35, 2013.

METZ, C. Essais sur la signification au cinéma, v.1. Paris: Klincksieck, 1971.

MORIN, É. Introduction à la pensée complexe. Paris, E.S.F, 1990.

MUCCHIELLI, A. Dictionnaire des méthodes qualitatives en sciences humaines. Paris: Armand Colin, 2009.

NORRIS, J. Playbuilding as Qualitative Research: A Participatory Arts-Based Approach. Walnut Creek, CA: Left Coast Press, 2009.

PASSERON, R. Recherches poḯtiques, n.1, 1975.

RABINOW, P. Reflections on fieldwork in Morocco. Berkeley: University of California Press (Quantum Book), 1977.

REMYSEN, W. Hétérogénéité et homogénéité dans les pratiques langagières: Mélanges offerts à Denise Deshaies. Québec: Les Presses de I'Université Laval, 2011. 
RICHARDSON, L. New writing practices in qualitative research. Sociology of sport journal, v.17, n.1, p. 5-20, 2000.

Richardson, L.; Adams, E. S. P. Writing: A Method of Inquiry. In DENZIN, N. K.; LINCOLN, Y. S. (eds.) The Sage Handbook of Qualitative Research. London: Sage, 2005, p. 959-78.

RICCEUR, P. Soi-même comme un autre. Paris: Seuil, 1990.

SAVOIE-ZAJC, L. Les critères de rigueur de la recherche qualitative/ interprétative : du discours à la pratique. Comunicação realizada no Colóquio l'Association pour la recherche qualitative: Regards actuels sur les critères de scientificité. Trois-Rivières, 28 novembro de 2003.

SCHAEFFER, J. M. Pourquoi la fiction?. Paris: Seuil, 1999.

SCHATZMAN, L.; STRAUSS, A.L. Field research. Strategies for a natural sociology. Englewood Cliffs: Prentice-Hall, 1973.

SCHÖN, D. A. Le tournant réflexif. Pratiques éducatives et études de cas. Montréal: Logiques, 1996.

TEDLOCK, D. The spoken word and the work of interpretation. Philadelphia: University of Pennsylvania Press, 1983.

TISSERON, S. La réalité de l'expérience de fiction. L'Homme 3/2005 (n 175-176), 2005.
VAN MAANEN, J. 1991. Quelques notes sur les normes et les formes d'écriture en sciences sociales. Recherches qualitatives, n. 5, 15-33, 1991.

VISWESARAN, K. Fictions of Feminist Ethnography. Minneapolis: University of Minnesota Press, 1994.

WEICK, K.E. Sensemaking in organizations, Thousand Oaks, CA: Sage, 1995.

WILLSON, M.; KULICK, D.; WILLSON, M. Afterword, perspective and difference: sexualization, the field, and the ethnographer. In KULICK, D.; WILLSON, M. (eds.). Taboo: sex, identity, and erotic subjectivity in anthropological fieldwork. Londres: Routledges, 1995, p. 251-277.

Recebido em: 15/09/2016 Aprovado em: 28/09/2016 